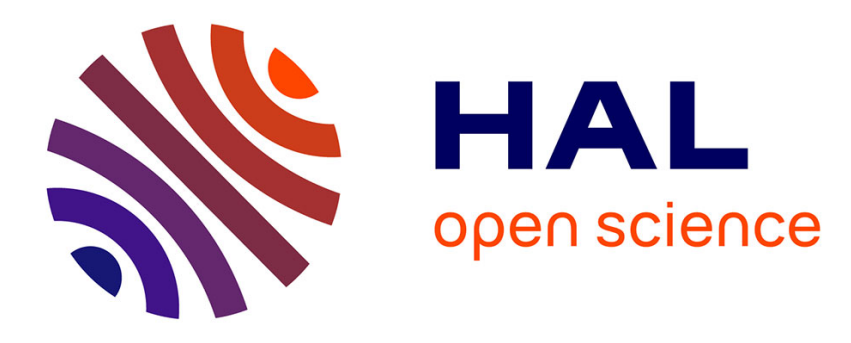

\title{
Concentration of Economic Activity: An Analytical Framework
}

\author{
Olga Alonso-Villar, Coral del Río
}

\section{To cite this version:}

Olga Alonso-Villar, Coral del Río. Concentration of Economic Activity: An Analytical Framework. Regional Studies, 2011, pp.1. 10.1080/00343404.2011.587796 . hal-00724178

\section{HAL Id: hal-00724178 \\ https://hal.science/hal-00724178}

Submitted on 19 Aug 2012

HAL is a multi-disciplinary open access archive for the deposit and dissemination of scientific research documents, whether they are published or not. The documents may come from teaching and research institutions in France or abroad, or from public or private research centers.
L'archive ouverte pluridisciplinaire HAL, est destinée au dépôt et à la diffusion de documents scientifiques de niveau recherche, publiés ou non, émanant des établissements d'enseignement et de recherche français ou étrangers, des laboratoires publics ou privés. 


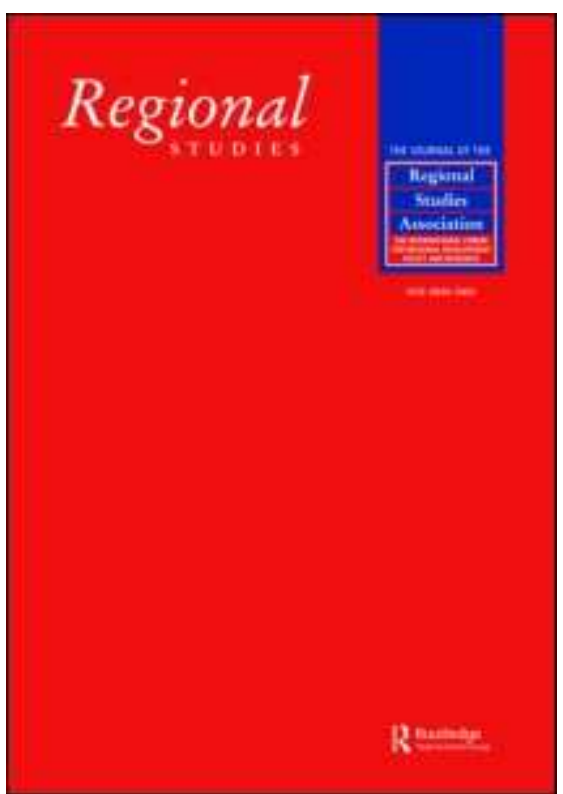

\section{Concentration of Economic Activity: An Analytical Framework}

\begin{tabular}{|r|l|}
\hline Journal: & Regional Studies \\
\hline Manuscript ID: & CRES-2010-0044.R2 \\
\hline Manuscript Type: & Main Section \\
\hline JEL codes: & $\begin{array}{l}\text { R12 - Size and Spatial Distributions of Regional Economic Activity < } \\
\text { Economics, D63 - Equity, Justice, Inequality, etc. and Measurement } \\
\text { < D6 - Welfare Economics < D - Microeconomics }\end{array}$ \\
\hline Keywords: & Geographic Concentration, Inequality Measures \\
\hline
\end{tabular}

\section{SCHOLARONE" Manuscripts}




\title{
Concentration of Economic Activity:
}

\section{An Analytical Framework}

\author{
Olga Alonso-Villar ${ }^{\#}$ and Coral del Río*
}

(Received February 2010: in revised form May 2011)

\footnotetext{
\# Universidade de Vigo; Facultade de CC. Económicas; Departamento de Economía Aplicada; Campus Lagoas-Marcosende s/n; 36310 Vigo; Spain. E-mail: ovillar@uvigo.es

* Universidade de Vigo; Facultade de CC. Económicas; Departamento de Economía Aplicada; Campus Lagoas-Marcosende s/n; 36310 Vigo; Spain. E-mail: crio@uvigo.es
} 


\begin{abstract}
The goal of this paper is to offer an analytical framework within which relative concentration, including both the concentration of each sector and aggregate concentration, can be analyzed. By borrowing properties from the literature on income inequality and segregation and adapting them to a location context, this paper characterizes the generalized entropy family of concentration indexes and shows the properties of the L-index. In addition, it offers other measures taken from the segregation literature. All these tools are used to analyze the spatial patterns of manufacturing industries in Spain from 1977 to 2008, paying special attention to their technological intensity.

JEL Classification: R12; D63
\end{abstract}

Keywords: Geographic concentration; Inequality measures 


\section{La Concentración de la Actividad Económica:}

\section{Un Marco Analítico}

\section{Resumen}

El objetivo de este trabajo es ofrecer un marco analítico dentro del cual examinar tanto la concentración relativa de un sector como la del conjunto de sectores. Tomando propiedades de la literatura de distribución de la renta y de segregación y adaptándolas a un contexto de localización, este artículo caracteriza las medidas de concentración derivadas de la familia de índices de entropía generalizada y muestra las propiedades del índice L. Además, ofrece también otras medidas derivadas de la literatura de segregación. Todas estas herramientas son usadas para analizar los patrones espaciales de la industrias manufactureras españolas entre 1977 y 2008, prestando especial atención a sus intensidades tecnológicas. 


\section{Introduction}

In recent years, the study of production location patterns has received increasing interest in the field, both empirically and theoretically. This flourishing interest is motivated in part by a general concern with the effects of economic integration processes on industrial localization, especially in Europe, where the creation of the Single Market has stimulated the debate (HAALAND et al., 1999; BRÜLHART, 2001; AIGINGER and PFAFFERMAYR, 2004; RESMINI, 2007, inter alia).

In quantifying the spatial concentration of a sector, most measures follow a relative notion so that the spatial distribution of the sector is compared with that of the whole set of sectors (ELLISON and GLAESER, 1997; AMITI, 1999; BRÜLHART and TRAEGER, 2005). If economic activity is measured in terms of employment, as is traditionally done, and the focus is on manufacturing industries, the distribution of overall manufacturing employment is usually considered the distribution of reference against which to compare that of any single sector. Thus, concentration rises in a sector as long as its employment distribution among locations departs from that of overall manufacturing employment.

By following this approach, many studies have calculated the concentration level of each manufacturing sector (which can be labeled partial concentration) in different economies; however, there has been no formal discussion of how to aggregate this information to calculate relative concentration for the entire manufacturing industry. Certainly, interest in measuring overall concentration is not new in the field, and complementary perspectives have been offered. Thus, some studies have proposed comparing distribution of employment in the manufacturing industry across locations with distribution of employment in the whole economy (BRÜLHART and TRAEGER, 2005). In other words, in measuring concentration in the manufacturing industry, the benchmark considered is outside that industry. Others have proposed instead the use of the average concentration of the sectors involved, making use of an external benchmark unnecessary (AIGINGER and DAVIS, 2004; MULLIGAN and SCHMIDT, 2005; CUTRINI, 2009, 2010). However, as far as we know, there has been no debate on overall concentration measurement in terms of basic properties. 
Among the spatial concentration measures existing in the literature, those borrowed from the literature on income inequality are some of the most widely used. In this regard, the Gini index has traditionally been used for analyzing spatial location patterns of manufacturing industries (KRUGMAN, 1991; AMITI, 1999; BRÜLHART, 2001; SUEDEKUM, 2006, inter alia). More recently, the generalized entropy family of indexes (GE, henceforth) has been used as well because of its advantages in terms of decomposability (BRÜLHART and TRAEGER, 2005; BRAKMAN et al., 2005; PÉREZ-XIMÉNEZ and SANZ-GRACIA, 2007; CUTRINI, 2009). The properties of the inequality measures on which these concentration indexes are based are well known because the literature on income distribution has dealt with inequality from an axiomatic perspective; however, the corresponding geographical concentration measures are being applied without explicitly stating the consequences of using them.

To close these gaps somewhat, this paper offers a set-up within which relative concentration, including both the concentration of each sector and aggregate concentration, can be analyzed in terms of basic properties. This set-up allows us to characterize the GE family of concentration indexes of a sector (BRÜLHART and TRAEGER, 2005) and show the properties of the aggregate concentration index, the Lindex (CUTRINI, 2009, 2010), resulting from the weighted sum of the one of them (the Theil index). For that purpose, some properties borrowed from the literature on income inequality and segregation are adapted to our context (ALONSO-VILLAR and DEL RÍO, 2010a; FRANKEL and VOLIJ, 2010). ${ }^{1}$ In addition, other partial and overall measures borrowed from the segregation literature are proposed to quantify concentration. All these tools are finally used to analyze the spatial patterns of manufacturing industries in Spain during its democratic period (1977-2008).

Our empirical analysis suggests that high-tech industries tend to concentrate at a higher extent than industries with a lower technological intensity. In addition, we find that overall concentration slighted increased until 1981 while it tended to decrease from 1985 onward. This evolution seems to be mainly shaped by concentration changes in the sectors involved and not by shifts in the sectoral structure of the economy.

The paper is structured as follows. Section 2 offers an overview of recent concentration measures and characterizes the partial concentration measures derived from the GE family in terms of basic properties. Section 3 introduces several aggregate concentration 
indexes related to the partial measures described above. These partial and aggregate measures are used in Section 4 to analyze the manufacturing industry in Spain, paying attention to the contribution of technological groups to overall concentration. Finally, Section 5 presents the main conclusions.

\section{The spatial concentration of an industry}

\subsection{An overview of recent concentration measures}

The literature offers a variety of measures to assess the distribution of economic activity across geographic units (counties, regions, countries, etc.). The terminology used is not always consistent since they are indiscriminately called spatial/geographical concentration measures, localization measures, and agglomeration measures. In this paper, we prefer the term geographical concentration, which seems more general, since the other labels are often used when these measures are intended to check the existence of externalities as the main force driving the spatial concentration of firms. ${ }^{2}$

Some of these measures are formally derived from location models, such as the popular index proposed by ELLISON and GLAESER (1997) (EG, henceforth), which measures the extent of geographic concentration once the size of establishments (via the Herfindahl index) and inherent randomness in the concentration of firms are accounted for. Following the same spirit, MAUREL and SÉDILLOT (1999) and GUIMARÃES et al. (2007) developed new indexes. Other indexes are instead borrowed from the literature on statistics, as is the case with the $D$-index proposed by MORI et al. (2005), which quantifies the divergence between the observed distribution of establishments and a uniform distribution across physical space according to the Kullback-Leibler distance (also called relative entropy). A similar index is that used by AIGINGER and DAVIES (2004) and AIGINGER and PFAFFERMAYR (2004), who borrowed the concept of entropy from information theory, rather than that of relative entropy (COVER and THOMAS, 1991). ${ }^{3}$ Other measures derived from the literature on statistics include those put forward by MARCON and PUECH (2003) and DURANTON and OVERMAN (2005). As opposed to previous measures, these tools are distance-based methods that consider the distribution of distances between pairs of establishments. This allows taking into account various spatial scales at the same time, 
avoiding the problem of arbitrary geographic scales (usually administrative). An important drawback of this approach is that it requires data that are often not available.

Apart from the EG index, which has been applied in a large number of empirical studies, some of the most widely used geographic concentration measures are derived from the literature on income inequality. This is the case with the Gini coefficient and the Theil index (also related to information theory). ${ }^{4}$ Several versions exist depending on the view of spatial concentration; thus, the absolute concentration adaptations of these measures are used when the no-concentration benchmark against which to compare the distribution of a sector is given by a uniform distribution across locations (BRAKMAN et al., 2005). There are also topographic concentration measures (BRAKMAN et al., 2005; BRÜLHART and TRAEGER, 2005) according to which noconcentration exists if the sector is evenly spread over physical space (which is also the approach followed in the $D$-index, which can actually be interpreted as the topographic version of the Theil index).

However, most concentration measures have followed a different approach. Suppose, for example, that economic activity is measured in terms of employment, as traditionally assumed. Focusing on manufacturing industries, the distribution of overall manufacturing employment is usually considered the distribution of reference against which to compare that of any single sector, so that no spatial concentration exists in the sector so long as its employment distribution across locations is equal to that of the industrial aggregate. This notion is labeled relative concentration and has been extensively used in empirical research (ELLISON and GLAESER, 1997; AMITI, 1999; BRÜLHART and TRAEGER, 2005). This is the approach followed in this paper when unveiling, in this section, the properties that scholars in regional science are implicitly assuming when using these inequality-based measures (which do not account for either inherent randomness in the concentration of firms or distances between locations).

The measures mentioned so far assess the concentration of a sector, labeled here partial concentration; however, one can also be concerned with aggregate or overall concentration. This issue will be analyzed in Section 3 . 


\subsection{Characterizing partial measures}

Since the literature on income distribution has tacked inequality from an axiomatic perspective, there is general agreement on the basic properties that any inequality measure should satisfy. The approach followed by the literature on geographic concentration has been rather different, since such an axiomatization does not exist (COMBES and OVERMAN, 2004). The aim of this paper is not to propose an axiomatic framework for measuring geographical concentration, but to unveil some of the main properties we implicitly assume when using inequality measures in this context.

For the sake of simplicity, assume that economic activity is measured in terms of employment. Consider that there are $T$ workers in the economy allocated among $L>1$ locations according to $t \equiv\left(t_{1}, t_{2}, \ldots, t_{L}\right)$, where $T=\sum_{l} t_{l}$. Vector $t$ represents the distribution of reference against which that of any sector is compared. Let us denote by $x^{s} \equiv\left(x_{1}^{s}, x_{2}^{s}, \ldots, x_{L}^{s}\right)$ the distribution of sector $s$, where $x_{l}^{s}$ is the number of workers in sector $s$ in location $l$. Therefore, the total number of workers in location $l$ is $t_{l}=\sum_{s} x_{l}^{s}$, while the total number of workers in sector $s$ is $X^{s}=\sum_{l} x_{l}^{s}$.

In this paper, an index of partial concentration is a function $I_{c}: D \rightarrow \square$, where $D=\bigcup_{L>1}\left\{\left(x^{s} ; t\right) \in \square^{L}{ }_{+} \times \square_{++}^{L}: x_{l}^{s} \leq t \forall l\right\}$, such that $I_{c}\left(x^{s} ; t\right)$ represents the concentration level of sector $s$, which is distributed across locations according to $x^{s}$, when comparing it with the distribution of reference $t$.

The GE family comprises indexes frequently used to measure the concentration of an industry according to a relative notion and can be written as:

$$
\Psi_{\alpha}\left(x^{s} ; t\right)=\left\{\begin{array}{l}
\frac{1}{\alpha(\alpha-1)} \sum_{l} \frac{t_{l}}{T}\left[\left(\frac{x_{l}^{s} / X^{s}}{t_{l} / T}\right)^{\alpha}-1\right] \text { if } \alpha \neq 0,1 \\
\sum_{l} \frac{x_{l}^{s}}{X^{s}} \ln \left(\frac{x_{l}^{s} / X^{s}}{t_{l} / T}\right) \text { if } \alpha=1
\end{array},\right.
$$


where $\alpha$ is a sensitivity parameter. ${ }^{5}$ If sector $s$ is distributed across locations in the same way as aggregate employment, i.e., if $x_{l}^{s} / X^{s}=t_{l} / T \quad \forall l$, any index of this class is equal to zero. ${ }^{6}$ An advantage of these measures is that they are additively decomposable, which is helpful for empirical analysis.

Even though in the literature on income distribution this family of indexes has been characterized in terms of basic properties, to our knowledge, such a characterization does not exist in the field of spatial concentration. In the next proposition, we show that five properties borrowed from the literature on inequality and segregation completely characterize the GE family of concentration indexes: ${ }^{7}$

1) Symmetry in locations (the partial concentration index is unaffected by the order in which locations are enumerated). This property implies that the spatial distribution of locations is irrelevant.

2) Movement between locations (when a location with a lower employment level in the sector of study than another, but with the same aggregate employment, loses employment in the sector in favor of the other location, the concentration of the sector must increase). This guarantees that a disequalizing movement of workers from one location to another having the same total employment level fosters concentration.

3) Scale invariance I (concentration should not change when the employment level of the aggregate distribution and/or that of the sector varies, so long as the weight that each location represents in distributions $t$ and $x^{s}, \frac{t_{l}}{T}$ and $\frac{x_{l}^{s}}{X^{s}}$, respectively, remains unaltered). This implies that it is only employment shares that matter, not employment levels.

4) Insensitivity to proportional divisions of locations I (subdividing a location into several units of equal size, in terms of both aggregate employment and employment in the sector of study, does not affect the concentration level of the sector). This property means that the geographical scale used in the analysis (usually administrative units) is irrelevant as long as employment distributions within locations are uniform. ${ }^{8}$

5) Aggregation (when classifying locations into two mutually exclusive classes, the concentration of the sector can be written as a function of the concentration of 
the sector in each class of locations, the employment level in each class, and the employment share of the sector in each class of locations). This is a helpful property since it allows the indexes to decompose by subgroups.

Proposition. Let $I_{c}$ be a continuous concentration index that takes a zero value when the distribution of the sector of study among locations coincides with the distribution of reference (i.e., when $\frac{x_{l}^{s}}{X^{s}}=\frac{t_{l}}{T}$ ). $I_{c}$ is a concentration index satisfying properties 1-5 if and only if it can be written as an increasing monotonic transformation of $\Psi_{\alpha}\left(x^{s} ; t\right)$.

Proof: See Appendix A.

Another relative measure widely used is the locational Gini coefficient:

$$
G^{s}=\frac{\sum_{l, l^{\prime}} \frac{t_{l}}{T} \frac{t_{l^{\prime}}}{T}\left|\frac{x_{l}^{s}}{t_{l}}-\frac{x_{l^{\prime}}^{s}}{t_{l^{\prime}}}\right|}{2 \frac{X^{s}}{T}},
$$

which satisfies properties 1-4 but not property 5. An additional discrepancy between $\Psi_{\alpha}$ and $G^{s}$ rests on their different sensitivity toward movements of workers between locations. Thus, $\Psi_{\alpha}$ (with $\alpha \leq 1$ ) is more sensitive to movements of workers between locations in which the relative presence of the sector, $\frac{x_{l}^{s}}{t_{l}}$, is low. ${ }^{9} G^{s}$ attaches, instead, more weight to movements affecting locations around the mode of distribution $\left(\frac{x_{1}^{s}}{t_{1}}, \ldots, \frac{x_{L}^{s}}{t_{L}}\right)$.

Given the parallels between spatial concentration of a sector across locations and segregation of a population group across organizational units, a variation of the index of dissimilarity proposed by DUNCAN and DUNCAN (1955) can be adapted to measure partial concentration:

$$
D^{s}=\frac{1}{2} \sum_{l}\left|\frac{x_{l}^{s}}{X^{s}}-\frac{t_{l}}{T}\right|
$$


It is easy to see that this index satisfies properties 1, 3, and 4, but not property 2 since $D^{s}$ is not sensitive to disequalizing movements of workers between locations on the same side of the mean of distribution $\left(\frac{x_{1}^{s}}{t_{1}}, \ldots, \frac{x_{L}^{s}}{t_{L}}\right)$. Therefore, as opposed to $\Psi_{\alpha}$ and $G^{s}, D^{s}$ does not always increase when disequalizing movements of workers occur. ${ }^{10}$

So far, we have focused on the properties analyzed in inequality/segregation contexts when using GE measures since, as far as we know, they have not been unveiled in a location context. These properties are of a different nature from those presented in COMBES and OVERMAN (2004). In any case, note that the GE family of concentration indexes also satisfies some of those properties. They are comparable across spatial scales, and some are also comparable across activities since they are decomposable by locations and subsectors, respectively (BRÜLHART and TRAEGER, 2005). In addition, statistical significance of the results is possible by using bootstrapping (BRÜLHART and TRAEGER, 2005) and the index takes a unique value (zero) under the null hypothesis of no concentration. Note also that all the partial measures included in this section are affected by the "modifiable areal unit problem" (MAUP, ARBIA, 1989), since in quantifying concentration they do not take into account the spatial distribution inside observed regions (as discussed by BRÜLHART and TRAEGER, 2005, in the GE case). This means that they are sensitive to changes in spatial scale. Moreover, since these measures neglect distances across regions, they are affected by the "checkerboard problem" as well (WHITE, 1983).

\section{Overall concentration measures}

In this section, we first show that the weighted sum of the Theil index $\left(\Psi_{1}\left(x^{s} ; t\right)\right)$ for each of the mutually exclusive sectors into which the economy can be partitioned (i.e., the $L$-index proposed by CUTRINI, 2009) is actually the mutual information index ( $M$, henceforth) derived from information theory. Next, by analyzing the properties satisfied by the $M$-index in other contexts, we unveil the properties that one is implicitly assuming when using it to measure geographic concentration. Finally, we propose two more aggregate concentration measures derived from the segregation literature to use in the empirical section to analyze the robustness of our results. 


\subsection{The $M$-index}

In a recent paper, CUTRINI (2009) proposes using the $L$-index, $L=\sum_{s} \frac{X^{s}}{T} \Psi_{1}\left(x^{s} ; t\right)$, to measure overall localization of manufacturing industries in Europe. Given that $\sum_{s} \frac{X^{s}}{T} \sum_{l} \frac{x_{l}^{s}}{X^{s}} \ln \left(\frac{t_{l}}{T}\right)=\sum_{l} \frac{\sum_{s} x_{l}^{s}}{T} \ln \left(\frac{t_{l}}{T}\right)=\sum_{l} \frac{t_{l}}{T} \ln \left(\frac{t_{l}}{T}\right)$, it is easy to see that this index can be rewritten as $L=\sum_{s} \frac{X^{s}}{T} \sum_{l} \frac{x_{l}^{s}}{X^{s}} \ln \left(\frac{x_{l}^{s}}{X^{s}}\right)-\sum_{l} \frac{t_{l}}{T} \ln \left(\frac{t_{l}}{T}\right)$. Note that, on the one hand the second component of the above expression is the entropy, $H(V)$, of a random variable, $V$, whose frequency distribution is $\left(\frac{t_{1}}{T}, \ldots, \frac{t_{L}}{T}\right)$. On the other hand, the first component is equal to minus the conditional entropy of $V \mid Z,-H(V \mid Z)$, where the distribution of $Z$ is $\left(\frac{X^{1}}{T}, \ldots, \frac{X^{S}}{T}\right)$, and the conditional distribution $V \mid Z=z$ is $\left(\frac{x_{1}^{s}}{X^{s}}, \ldots, \frac{x_{L}^{s}}{X^{s}}\right)$. From all of the above, it follows that the $L$-index turns out to be equal to the $M$-index of random distributions $V$ and $Z$, since $M$ is defined as $M(V ; Z)=H(V)-H(V \mid Z), \quad$ where $\quad H(V)=-\sum_{v} p(v) \ln p(v)$, $H(V \mid Z)=-\sum_{z} p(z) \sum_{v} p(v \mid z) \ln p(v \mid z), \quad p(v)$ and $p(z)$ denote the probability mass functions of $V$ and $Z$, respectively, and $p(v \mid z)$ is the probability distribution of conditional distribution $V \mid Z=z$ (see COVER and THOMAS, 1991). In other words,

$$
L=\underbrace{-\sum_{l} \frac{t_{l}}{T} \ln \left(\frac{t_{l}}{T}\right)}_{H(V)}+\underbrace{\sum_{s} \frac{X^{s}}{T} \sum_{l} \frac{x_{l}^{s}}{X^{s}} \ln \left(\frac{x_{l}^{s}}{X^{s}}\right)}_{-H(V \mid Z)}=M(V ; Z) .
$$

Therefore, $M$ can also be written as

$$
M=\sum_{s} \frac{X^{s}}{T} \Psi_{1}\left(x^{s} ; t\right) .
$$

The $M$-index has been axiomatically characterized by FRANKEL and VOLIJ (2010) for analyzing overall school segregation in a multiracial context. ${ }^{11}$ The parallels between 
measurement of overall segregation across organizational units and measurement of overall geographic concentration is evident. The former involves comparisons among the distributions of racial groups across schools, while the latter requires comparing distributions of industries across locations. Given the good properties of this index in a segregation context (actually considered axioms, see JAMES and TAEUBER, 1985), it also seems reasonable to analyze its suitability for quantifying overall concentration.

In what follows, we adapt these properties to our context (for more technical definitions, see Appendix A). Some of these properties are similar to those previously defined for partial measures except that now all sectors are involved, which implies adding specific requirements for them.

The $M$-index satisfies continuity and is invariant to:

a) Any reordering of sectors and locations (symmetry in sectors and locations);

b) Proportional changes in all sectors and locations (scale invariance II);

c) Splitting one location into two if both have the same sectoral structure (insensitivity to proportional divisions of locations II).

In addition,

d) If a sector is split into two subsectors both having the same distribution across locations, concentration remains unaltered (group division). This implies that concentration is unbiased to the industrial classification so long as there are no internal differences among the spatial distributions of subsectors included in each sector.

e) Given two economies having the same total employment and sizes of sectors (locations), if an economy, representing new locations (sectors), is adjoined to each of them, the ranking between them according to the index does not change (independence). This is a separability property that means that if workers of a sector (location) are reallocated among a subset of locations (sectors), concentration rises if and only if concentration in these locations (sectors) rises.

An advantage of this index is that it can be decomposed additively by groups (CUTRINI, 2009). Thus, if the manufacturing sectors are classified into several mutually exclusive groups, it is possible to determine whether aggregate manufacturing concentration is due mainly to the between-group $(B)$ component (i.e., to differences in the spatial distribution of employment between these groups) or to the within-group $(W)$ 
component (i.e., to differences in the spatial distributions of the sectors included in each group compared with employment distribution of the whole group). Let us assume that the manufacturing sectors are classified into (say) two groups: low-tech $(G)$ and hightech industries $(H)$. Then,

$$
M=M^{B}+M^{W}
$$

where:

$$
\begin{gathered}
M^{B}=\frac{X^{G}}{T} \sum_{l} \frac{x_{l}^{G}}{X^{G}} \ln \left(\frac{\frac{x_{l}^{G}}{X^{G}}}{\frac{t_{l}}{T}}\right)+\frac{X^{H}}{T} \sum_{l} \frac{x_{l}^{H}}{X^{H}} \ln \left(\frac{\frac{x_{l}^{H}}{X^{H}}}{\frac{t_{l}}{T}}\right), \text { and } \\
M^{W}=\frac{X^{G}}{T} \sum_{s \in G} \frac{X^{s}}{X^{G}} \sum_{l} \frac{x_{l}^{s}}{X^{s}} \ln \left(\frac{\frac{x_{l}^{s}}{X^{s}}}{\frac{x_{l}^{G}}{X^{G}}}\right)+\frac{X^{H}}{T} \sum_{s \in H} \frac{X^{s}}{X^{H}} \sum_{l} \frac{x_{l}^{s}}{X^{s}} \ln \left(\frac{\frac{x_{l}^{s}}{X^{s}}}{\frac{x_{l}^{H}}{X^{H}}}\right),
\end{gathered}
$$

where $x_{l}^{G}\left(x_{l}^{H}\right)$ represents the low-tech (high-tech) employment in location $l$, and $X^{G}$ $\left(X^{H}\right)$ is the total low-tech (high-tech) employment in the economy.

The $M$-index can also be used to compare overall concentration in two different years. To do that, let us calculate the weighted average of index $\Psi_{1}$ in year 1 according to demographic weights in year 2: $\sum_{s} \frac{X^{s(2)}}{T^{(2)}} \Psi_{1}\left(x^{s(1)} ; t^{(1)}\right)$. Next, add and subtract that expression in $M^{(2)}-M^{(1)}$. It is straightforward to show that an intertemporal change between years (2) and (1) can be decomposed in two terms, one showing the gap due to changes in the spatial concentration of sectors and another due to changes in the sectoral structure of the economy:

$$
M^{(2)}-M^{(1)}=\underbrace{\sum_{s} \frac{X^{s(2)}}{T^{(2)}}\left(\Psi_{1}\left(x^{s(2)} ; t^{(2)}\right)-\Psi_{1}\left(x^{s(1)} ; t^{(1)}\right)\right)}_{\text {concentration factor }}+\underbrace{\sum_{s} \Psi_{1}\left(x^{s(1)} ; t^{(1)}\right)\left(\frac{X^{s(2)}}{T^{(2)}}-\frac{X^{s(1)}}{T^{(1)}}\right)}_{\text {sectoral structure factor }} .
$$

The first component (concentration factor) aggregates the differences between concentration index $\Psi_{1}$ in years 2 and $1\left(\Psi_{1}\left(x^{s(2)} ; t^{(2)}\right)-\Psi_{1}\left(x^{s(1)} ; t^{(1)}\right)\right)$ according to the demographic weights in year 2. The second component (sectoral structure factor) aggregates the differences due to changes in the demographic weight of each sector $\left(\left(X^{s(2)} / T^{(2)}\right)-\left(X^{s(1)} / T^{(1)}\right)\right)$. 
$M$ also satisfies several of the properties discussed in COMBES and OVERMAN (2004), as mentioned in CUTRINI (2009) with respect to the $L$-index. The withinbetween decomposition allows $M$ to be comparable across activities at different scales. ${ }^{12}$ In addition, since $M$ is equal to the weighted average of $\Psi_{1}$, which is decomposable by groups of locations, $M$ is also comparable across spatial scales. For the same reason, statistical significance of the results is possible by using bootstrapping, and the index takes a unique value (zero) under the null hypothesis of no concentration.

\subsection{Other overall concentration indexes}

The unbounded Gini index, $G$, proposed by REARDON and FIREBAUGH (2002) to measure overall segregation can also be used to quantify aggregate concentration since it can be expressed as the weighted mean of index $G^{s}$ for each sector:

$$
G=\sum_{s} \frac{X^{s}}{T} G^{s}
$$

SILBER (1992) also offers an overall segregation index that extends the popular index of dissimilarity proposed by DUNCAN and DUNCAN (1955). This index can be adapted to measure overall concentration as follows:

$$
I S=\frac{1}{2} \sum_{s} \sum_{l}\left|\frac{x_{l}^{s}}{T}-\frac{X^{s}}{T} \frac{t_{l}}{T}\right| .
$$

It is easy to prove that this modified version can also be written as the weighted sum of index $D^{s}$ for each sector into which the manufacturing industry can be partitioned:

$$
I S=\sum_{s} \frac{X^{s}}{T} D^{s}
$$

Consequently, $G$ and $I S$ are naturally related to the aforementioned partial measures since they are weighted averages of $G^{s}$ and $D^{s}$, respectively. ${ }^{13}$ This implies, first, that they can be used to determine the contribution of each sector to overall concentration, as happens in the case of $M$. And second, the differences among $M, G$, and $I S$ rest on the disparities among the partial measures on which they are based. These indexes can also be decomposed like the $M$-index to undertake intertemporal comparisons.

In the empirical section, we show not only the results with the $M$-index but also with the above indexes. This allows us to check the robustness of our results since $M, G$, and $I S$ 
are based on partial concentration measures satisfying different properties (as shown in Section 2.2), which means that these indexes do not necessarily lead to the same outcomes.

\section{Concentration of manufacturing industries in Spain: An illustration}

This section illustrates the use of the above tools in the case of Spain, showing the usefulness of the decompositions of these indexes both by groups of sectors (classified by technological intensity) and across time. The data used in this paper come from the Labor Force Survey (EPA) conducted by the Spanish Institute of Statistics (INE) following EUROSTAT guidelines. Our data correspond to the second quarter of each year from 1977 to 2008. Manufacturing industries are considered at a two-digit level in the National Classification of Economic Activities (CNAE), and the territorial scale is that of provinces (nuts III).

\subsection{Manufacturing concentration in 2008 by technological intensity}

When analyzing spatial patterns of manufacturing industries in 2008, we find important similarities in the rankings of these industries according to partial indexes $\Psi_{1}\left(x^{s} ; t\right)$, $G^{s}$, and $D^{s} .{ }^{14}$ Moreover, all these indexes coincide in detecting concentration in both low-tech industries at a two-digit level (tobacco, leather, and recycling) and high-tech industries (office and IT equipment; aircraft and other transport material).

To analyze in more detail whether substantial differences exist among industries depending on their technological intensity, we group manufacturing industries by following the OECD and INE classifications (four groups of sectors have been considered, see Table 5 in Appendix B). Thus, we calculate the contribution of each sector to the overall concentration at a two-digit level and then aggregate these contributions according to the technology group to which each sector belongs. Three overall measures are used to obtain the concentration of the whole manufacturing industry: $M, G$, and $I S$ (see Table 1 and expressions (5)-(7)). ${ }^{15}$ Note that the contribution of a group of industries to overall concentration depends on two factors: the concentration of its industries and the employment share of the group. If the contributions of all groups are similar to their demographic weights, one can conclude 
that there are barely differences among the concentration levels of the groups. So long as the contribution of a group is higher than its demographic weight, the concentration of the group is rather intense (and, therefore, higher than that of other groups). The opposite happens when the contribution is lower than its demographic weight.

We find that the contribution of high-tech industries to overall concentration when using $M$ more than doubles their demographic weight (12.8\% versus $5.1 \%)$ and it also exceeds their demographic weight, although at a lower extent, when using $G$ and $I S$ (8.6\% versus $5.1 \%$ ). Regarding medium-high-tech industries, the results do depend on the index being used. On the contrary, in the case of medium-low-tech industries, their contribution to overall concentration is remarkably lower than expected (21-22\% versus $27 \%$ ) while the contribution of low-tech sectors to overall concentration is similar to the weight this group represents in terms of manufacturing employment.

\section{[Insert Table 1 around here]}

Consequently, high-tech industries seem more concentrated than industries with a lower technological intensity; however, can we say that technological intensity is a relevant variable to explain concentration in Spain? To answer this, we use the corresponding within-between decomposition of index $M$. We find that the between-group component explains around $28.5 \%$ of overall manufacturing concentration at a two-digit level. Therefore, there are indeed important differences in the spatial distributions of these four groups. Moreover, concentration increases with technological intensity (see Table 2). Thus, index $\Psi_{1}$ indicates that partial concentration in the high-tech group (jointly considered) triples that of the medium-high-tech group, while that of the latter doubles the value of the groups with lower technological intensity. ${ }^{16}$ Consequently, the spatial pattern of the high-tech group considered as a whole (i.e., neglecting the disparities among its sectors) clearly departs from the spatial distribution of total employment, which leads to a high concentration level.

[Insert Table 2 around here]

From all of the above, it seems that the technological intensity of industries plays an important role in explaining the concentration of manufacturing employment in Spain since the high-tech industries, jointly considered, have a higher concentration value than the remaining groups. This result is in line with that obtained by ALONSO-VILLAR et 
al. (2004), who used the index proposed by MAUREL and SÉDILLOT (1999) and a different Spanish dataset for 1999. Therefore, the results for the Spanish economy seem rather robust.

\subsection{Evolution of manufacturing concentration, 1977-2008}

Figure 1 shows the evolution of the overall concentration of the manufacturing industry at a two-digit level from 1977 to $2008 .{ }^{17}$ We can establish three periods of change in the evolution of spatial concentration. First, we observe a slight increase until 1981, a noteworthy decrease from 1985 to 1990, and finally, a remarkable decline from 2001 onward. ${ }^{18}$ The bootstrap analysis for the $M$ index based on 10,000 replications shows that the three changes are significant at the $99 \%$ level of significance (the null hypothesis being $\Delta M=0) .{ }^{19}$

\section{[Insert Figure 1 around here]}

By using the temporal decompositions of the above overall measures (Table 3), we find that the overall concentration rise in the first years of democracy is due entirely to the increase in concentration of the manufacturing industry (concentration factor). Decreases in the other two periods are also due mainly to changes in the concentration levels of the manufacturing industries (at least $60 \%$ of total change), even though the sectoral structure factor has an important weight, as well.

[Insert Table 3 around here]

[Insert Figure 2 around here]

Figure 2 shows the contributions of industries, grouped by technological intensity, to overall concentration at a two-digit level throughout the entire period. ${ }^{20}$ We observe a decreasing tendency in the contribution of low-tech industries to overall concentration from 1999 onward, together with an increase in the contribution of medium-high-tech industries from 2004 onward. We should note, however, that while the decrease in the low-tech group could be explained by the evolution of employment in this group, the evolution of the medium-high-tech group seems to be explained only partially by that factor (see Figure 3 in Appendix B). 


\section{Final comments}

The measurement of population segregation across organizational units (occupations, schools, neighborhoods, etc.) and the measurement of spatial concentration share much in common. Thus, while the former focuses on the distribution of racial groups across schools, for example, the latter addresses the distribution of economic sectors across location units; however, each field has dealt with measurement from a different perspective. On the one hand, while the segregation literature has tackled this matter mainly from an axiomatic point of view, the literature on spatial concentration has not. On the other hand, the former has focused mostly on the measurement of overall segregation, whereas the latter has dealt with the concentration of any single sector (labeled here partial concentration) rather than with overall concentration; exceptions are AIGINGER and DAVIES (2004), MULLIGAN and SCHMIDT (2005), and CUTRINI $(2009,2010)$.

Given the parallels between the two phenomena, this paper has proposed two overall concentration measures which are adapted from segregation measures (REARDON and FIREBAUGH, 2002, and SILBER, 1992). In addition, using FRANKEL and VOLIJ's (2010) characterization of the mutual information index, used to quantify school segregation, this paper has shown the properties of the L-index proposed by CUTRINI (2009). The partial concentration index in which the L-index is based, the Theil index, together with the remaining members of the GE family of concentration indexes, has been also characterized in terms of basic properties adapted from the literature on income distribution and segregation to our case. Consequently, this paper brings analytical support to partial and overall concentration measures existing in the literature (BRÜLHART and TRAEGER, 2005; CUTRINI, 2009, 2010).

Finally, these indexes have been used to measure overall concentration of Spanish manufacturing industries over the last three decades. The analysis reveals that the process of economic integration of Spain into the EU, signed in 1986, together with improvements in transport infrastructure, has not fueled the spatial concentration of the Spanish manufacturing industry. On the contrary, this process seems to be accompanied by a decreasing, though intermittent, trend in the concentration level that continues today. We have also found that the technological intensity of an industry is a relevant variable to explain geographic concentration in Spain. 


\section{Acknowledgements}

Financial support from the Xunta de Galicia (INCITE08PXIB300005PR) and from FEDER is gratefully acknowledged.

\section{Appendix A}

\section{Properties of partial measures}

Symmetry in locations. If $\left(x^{s} ; t^{\prime}\right)$ is obtained from $\left(x^{s} ; t\right)$ by a permutation of locations, then $I_{c}\left(x^{s} ; t^{\prime}\right)=I_{c}\left(x^{s} ; t\right)$.

Movement between locations. If $\left(x^{s} ; t\right)$ is obtained from $\left(x^{s} ; t\right)$ in such a way that: (i) $x_{i}^{s{ }^{\prime}}=x_{i}^{s}-d, x_{h}^{s{ }^{\prime}}=x_{h}^{s}+d \quad\left(0<d \leq x_{i}^{s}\right)$, where $i$ and $h$ are two locations satisfying that $t_{i}=t_{h}$, and $x_{i}^{s}<x_{h}^{s}$; and (ii) $x_{l}^{s}{ }^{\prime}=x_{l}^{s} \forall l \neq i, h$; then $I_{c}\left(x^{s} ; t\right)>I_{c}\left(x^{s} ; t\right)$.

Scale invariance I. If $a, b \in R^{++}\left(a x_{l}^{s} \leq b t_{l}\right)$, then $I_{c}\left(a x^{s} ; b t\right)=I_{c}\left(x^{s} ; t\right)$.

Insensitivity to proportional subdivisions of locations I. If $\left(x^{\prime} ; t^{\prime}\right)$ is obtained from $\left(x^{s} ; t\right)$ after splitting location $l$ into two in such a way that the size of the sector in each of them is $x_{l} / 2$ and the size of the distribution of reference in each of them is $t_{l} / 2$, then $I_{c}\left(x^{s} ; t^{\prime}\right)=I_{c}\left(x^{s} ; t\right)$.

Aggregation. Let us classify locations into two mutually exclusive groups so that $\left(x^{s} ; t\right)=\left(x^{s 1}, x^{s 2} ; t^{1}, t^{2}\right)$, where the aggregate employment level in locations included in group 1 (2) is denoted by $T_{1}\left(T_{2}\right)$, while $X_{1}^{s}\left(X_{2}^{s}\right)$ represents the employment level of the sector of study in the corresponding group of locations. Concentration index $I_{c}$ is defined as aggregative if there exists a continuous aggregator function $A$ such that $I_{c}\left(x^{s} ; t\right)=A\left(I_{c}\left(x^{s 1} ; t^{1}\right), \frac{X_{1}^{s}}{T_{1}}, T_{1}, I_{c}\left(x^{s 2} ; t^{2}\right), \frac{X_{2}^{s}}{T_{2}}, T_{2}\right)$, where $A$ is strictly increasing in the first and fourth arguments. 


\section{Properties of overall measures}

In order to formally define the corresponding properties of overall measures, we denote overall index by $O I_{c}$ and define matrix $E$ :

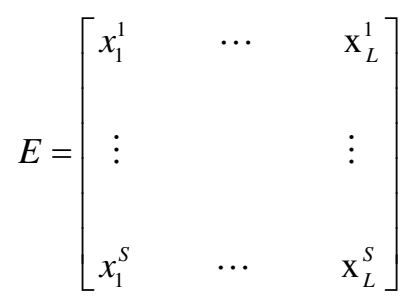

Symmetry in sectors and locations. If $E^{\prime}$ is obtained from $E$ by a permutation of sectors/locations, then $O I_{c}\left(E^{\prime}\right)=O I_{c}(E)$.

Scale invariance II. If the size of all sectors in all locations is multiplied by $\lambda \in R^{++}$, overall concentration does not change, i.e., $O I_{c}(\lambda E)=O I_{c}(E)$.

Insensitivity to proportional subdivisions of locations II. If matrix $E^{\prime}$ results from $E$ after splitting a location $l$ into two in such a way that the proportion of each sector $s$ (with respect to $t$ ) in each new location is the same, then $O I_{c}(E)=O I_{c}\left(E^{\prime}\right)$.

Group division. If $E^{\prime}$ results from $E$ by splitting sector $s$ into two subsectors, $s^{\prime}$ and $s^{\prime \prime}$, such that both have the same spatial distribution $\left(\frac{x_{l}^{s^{\prime}}}{X^{s^{\prime}}}=\frac{x_{l}^{s^{\prime \prime}}}{X^{s^{\prime \prime}}} \forall l\right)$, then $O I_{c}\left(E^{\prime}\right)=O I_{c}(E)$.

Independence. Consider two economies, $E$ and $\tilde{E}$, having the same total employment and the same employment level in each sector (location). If $E^{\prime}$ is obtained from $E$ and $\tilde{E}^{\prime}$ from $\tilde{E}$ after adjoining to each of them a common economy with new locations (sectors), then $O I_{c}\left(E^{\prime}\right) \geq O I_{c}\left(\tilde{E}^{\prime}\right)$ if and only if $O I_{c}(E) \geq O I_{c}(\tilde{E})$.

\section{Proof of proposition}

In the first step, we establish a formal relationship between geographic concentration and inequality (i.e., we show that any concentration index $I_{c}$ satisfying properties 1-4 leads to a inequality index, $I$, satisfying scale invariance, symmetry, the Pigou-Dalton 
principle, and replication invariance). In the second step, we make use of this relation, together with the results from the inequality literature, to show that any concentration index satisfying properties $1-5$ is a strictly increasing monotonic transformation of $\Psi_{\alpha}$. In the third step, we show that any strictly increasing monotonic transformation of $\Psi_{\alpha}$ satisfies properties $1-5$.

\section{First step.}

If the concentration index $I_{c}$ satisfies properties $1-4$, then index $I$ evaluated at the hypothetical distribution $y \equiv(\underbrace{\frac{x_{1}^{s}}{t_{1}}, \ldots, \frac{x_{1}^{s}}{t_{1}}}_{t_{1}}, \ldots, \underbrace{\frac{x_{L}^{s}}{t_{L}}, \ldots, \frac{x_{L}^{s}}{t_{L}}}_{t_{L}})$ as $I(y):=I_{c}\left(x^{s} ; t\right)$ works as an inequality index since it satisfies scale invariance, symmetry, the Pigou-Dalton principle, and replication invariance (these are axioms proposed in the literature on income distribution and we maintain their original labels).

a) $I$ is well defined. Note that several vectors $\left(x^{s} ; t\right)$ can be reached after grouping individuals in the hypothetical distribution who belong to the same location depending on how many locations are considered. However, by property 4, all of these vectors have the same spatial concentration level since they can be obtained from each other by proportional subdivisions.

b) Scale invariance. This property is satisfied by index $I$ since $I\left(\theta \frac{x_{1}^{s}}{t_{1}}, \ldots, \theta \frac{x_{1}^{s}}{t_{1}}, \ldots, \theta \frac{x_{L}^{s}}{t_{L}}, \ldots, \theta \frac{x_{L}^{s}}{t_{L}}\right)=I_{c}\left(\theta x^{s} ; t\right)$, which is equal to $I_{c}\left(x^{s} ; t\right)$ because $I_{c}$ satisfies property 3 (case where $a>0, b=1$ ).

c) Symmetry. It requires that individuals play symmetric roles in the inequality index. This is satisfied by $I$ since $I_{c}$ satisfies properties 1 and 4 .

d) The Pigou-Dalton transfer principle. According to property 4, any regressive transfer in this hypothetical economy can be expressed as a sequence of disequalizing employment movements in an economy constructed from the original one by proportional subdivisions of locations so that the distribution of 
reference becomes $(\underbrace{1, \ldots, 1}_{T})$. Since $I_{c}$ satisfies property 2 , the second situation leads to a higher concentration index and, therefore, to a higher value of $I$.

e) Replication invariance. It means that when replicating the economy k-times so that for every individual in the previous economy there are now $k$ identical individuals, income inequality is not altered. This property is satisfied here since a k-replication of the hypothetical distribution leads to a k-replication of vector $\left(x^{s} ; t\right)$, and $I_{c}$ satisfies property 3 (case where $a=b$ ).

\section{Second step.}

Following SHORROCKS (1984) and FOSTER (1985), any continuous inequality measure $I$ taking a zero value at the egalitarian distribution and satisfying scale invariance, replication invariance, the Pigou-Dalton transfer principle, symmetry, and aggregation can be written as $I(y)=F^{-1}\left(I_{\alpha}(y)\right)$ for some parameter $\alpha$, where $F$ is a strictly increasing function such that $F:[0, \infty) \rightarrow \square$ with $F(0)=0$ and $I_{\alpha}$ is the wellknown GE family of inequality indexes:

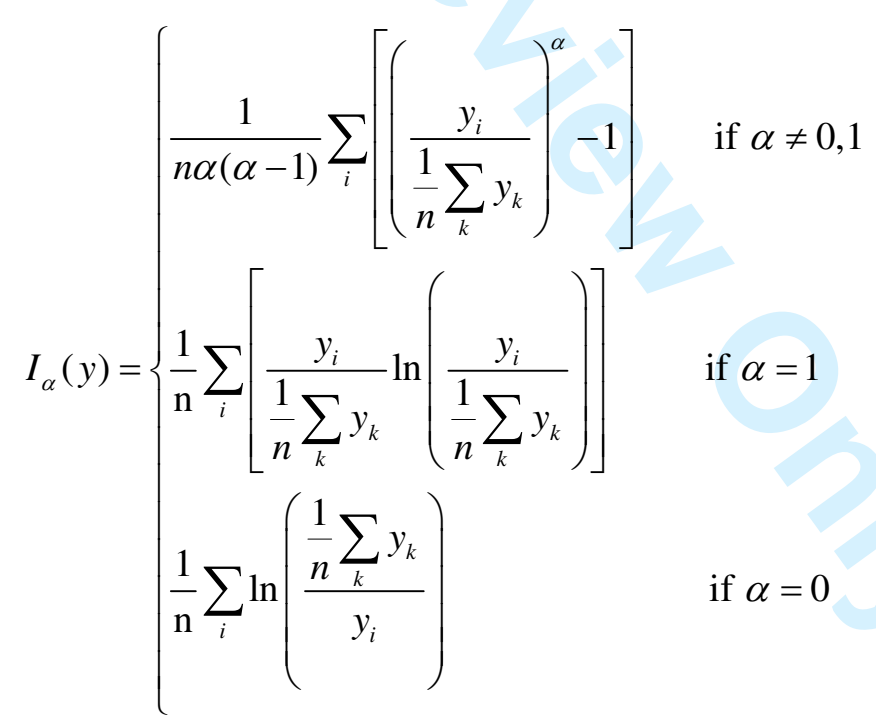

In Step 1, we proved that any concentration index $I_{c}$ satisfying properties 1-4 can be regarded as an inequality index $I$ satisfying scale invariance, symmetry, the PigouDalton transfer principle and replication invariance. It is easy to see that if $I_{c}$ is a continuous function, so too is $I$. If we additionally show that $I$ is aggregative and also 
that it is equal to zero at the egalitarian distribution, we can use Shorrocks's result to characterize inequality index $I$.

An inequality index $I$ is defined as aggregative if $I(y)=A\left(I\left(y^{1}\right), \mu\left(y^{1}\right), n\left(y^{1}\right), I\left(y^{2}\right), \mu\left(y^{2}\right), n\left(y^{2}\right)\right)$, where $A$ is a continuous function that is strictly increasing in the first and fourth arguments, $y^{i}$ represents the income distribution corresponding on the individuals' group $i, \mu($.$) is the average of the$ corresponding distribution, and $n($.$) is the number of individuals in the corresponding$ group. In our case, $y \equiv(\underbrace{\frac{x_{1}^{s}}{t_{1}}, \ldots, \frac{x_{1}^{s}}{t_{1}}}_{t_{1}}, \ldots, \underbrace{\left.\frac{x_{L}^{s}}{t_{L}}, \ldots, \frac{x_{L}^{s}}{t_{L}}\right)}_{t_{L}}$, and the average of that distribution is equal to $\frac{X^{s}}{T}$. In what follows, we show that our $I$ is an aggregative inequality index. For the sake of simplicity, assume that class 1 includes locations $l=1, \ldots, i$, while class 2 is the complementary. By definition

$$
I(\underbrace{\frac{x_{1}^{s}}{t_{1}}, \ldots, \frac{x_{1}^{s}}{t_{1}}, \ldots, \frac{x_{i}^{s}}{t_{i}}, \ldots, \frac{x_{i}^{s}}{t_{i}}}_{\text {class } 1} \underbrace{\frac{x_{i+1}^{s}}{t_{i+1}}, \ldots, \frac{x_{i+1}^{s}}{t_{i+1}}, \ldots, \frac{x_{L}^{s}}{t_{L}}, \ldots, \frac{x_{L}^{s}}{t_{L}}}_{\text {class } 2})=I_{c}\left(x^{s} ; t\right) .
$$

According to property $5, I_{c}$ is an aggregative concentration index:

$$
I_{c}\left(x^{s} ; t\right)=I_{c}\left(x^{s 1}, x^{s 2} ; t^{1}, t^{2}\right)=A\left(I_{c}\left(x^{s 1} ; t^{1}\right), \frac{X_{1}^{s}}{T_{1}}, T_{1}, I_{c}\left(x^{s 2} ; t^{2}\right), \frac{X_{2}^{s}}{T_{2}}, T_{2}\right)
$$

Note that $I_{c}\left(x^{s 1} ; t^{1}\right)=I\left(\frac{x_{1}^{s}}{t_{1}}, \ldots, \frac{x_{1}^{s}}{t_{1}}, \ldots, \frac{x_{i}^{s}}{t_{i}}, \ldots, \frac{x_{i}^{s}}{t_{i}}\right), \quad$ and $\quad I_{c}\left(x^{s 2} ; t^{2}\right)=I\left(\frac{x_{i+1}^{s}}{t_{i+1}}, \ldots, \frac{x_{i+1}^{s}}{t_{i+1}}, \ldots, \frac{x_{L}^{s}}{t_{L}}, \ldots, \frac{x_{L}^{s}}{t_{L}}\right)$

Therefore, the inequality index $I$ is aggregative:

$$
I(\underbrace{\frac{x_{1}^{s}}{t_{1}}, \ldots, \frac{x_{1}^{s}}{t_{1}}, \ldots, \frac{x_{i}^{s}}{t_{i}}, \ldots, \frac{x_{i}^{s}}{t_{i}}}_{\text {class } 1} \underbrace{\frac{x_{i+1}^{s}}{t_{i+1}}, \ldots, \frac{x_{i+1}^{s}}{t_{i+1}}, \ldots, \frac{x_{L}^{s}}{t_{L}}, \ldots, \frac{x_{L}^{s}}{t_{L}}}_{\text {class } 2})=A\left(I\left(\frac{x_{1}^{s}}{t_{1}}, \ldots, \frac{x_{1}^{s}}{t_{1}}, \ldots, \frac{x_{i}^{s}}{t_{i}}, \ldots, \frac{x_{i}^{s}}{t_{i}}\right), \frac{X_{1}^{s}}{T_{1}}, T_{1}, I\left(\frac{x_{i+1}^{s}}{t_{i+1}}, \ldots, \frac{x_{i+1}^{s}}{t_{i+1}}, \ldots, \frac{x_{L}^{s}}{t_{L}}, \ldots, \frac{x_{L}^{s}}{t_{L}}\right), \frac{X_{2}^{s}}{T_{2}}, T_{2}\right) .
$$

Finally, note that $I$ is equal to zero at the equalitarian distribution (i.e., when all locations have the same employment shares in the sector). 
Therefore, by using Shorrocks's result, it follows that $I(y)=F^{-1}\left(I_{\alpha}(y)\right)$ for $\alpha \neq 0,1$ or $\alpha=1 .^{21}$ On the other hand, $I_{c}\left(x^{s} ; t\right)=I(y)$ and $F^{-1}\left(I_{\alpha}(y)\right)=F^{-1}\left(\Psi_{\alpha}\left(x^{s} ; t\right)\right)$, which completes the proof of step two.

\section{Third step.}

To prove that $F^{-1}\left(\Psi_{\alpha}\right)$ is a concentration index satisfying properties $1-5$, it suffices to show that $\Psi_{\alpha}$ satisfies them, which is done in what follows. It is easy to prove that $\Psi_{\alpha}$ verifies scale invariance I, symmetry in locations, and insensitivity to proportional subdivisions I. To demonstrate that $\Psi_{\alpha}$ satisfies the property of movement between locations, note that any disequalizing movement from location $i$ to $h$, where $t_{i}=t_{h}$ and $x_{i}^{s}<x_{h}^{s}$, implies moving from distribution $y=\left(\frac{x_{1}^{s}}{t_{1}}, \ldots, \frac{x_{1}^{s}}{t_{1}}, \ldots, \frac{x_{i}^{s}}{t_{i}}, \ldots, \frac{x_{i}^{s}}{t_{i}}, \ldots, \frac{x_{h}^{s}}{t_{h}}, \ldots, \frac{x_{h}^{s}}{t_{h}}, \ldots, \frac{x_{L}^{s}}{t_{L}}, \ldots, \frac{x_{L}^{s}}{t_{L}}\right) \quad$ to $\quad$ distribution $y^{\prime}=\left(\frac{x_{1}^{s}}{t_{1}}, \ldots, \frac{x_{1}^{s}}{t_{1}}, \ldots, \frac{x_{i}^{s}-d}{t_{i}}, \ldots, \frac{x_{i}^{s}-d}{t_{i}}, \ldots \frac{x_{h}^{s}+d}{t_{h}}, \ldots, \frac{x_{h}^{s}+d}{t_{h}}, \ldots, \frac{x_{L}^{s}}{t_{L}}, \ldots, \frac{x_{L}^{s}}{t_{L}}\right) . \quad$ Note $\quad$ that $I_{\alpha}(y)=\Psi_{\alpha}\left(x^{s} ; t\right)$ and $I_{\alpha}\left(y^{\prime}\right)=\Psi_{\alpha}\left(x^{s} ; t^{\prime}\right)$. Since $I_{\alpha}$ is an inequality measure satisfying the Pigou-Dalton transfer principle and $y$ ' can be obtained from $y$ by a finite sequence of regressive transfers it follows that $\Psi_{\alpha}\left(x^{s} ; t^{\prime}\right)>\Psi_{\alpha}\left(x^{s} ; t\right)$.

Next, we prove that $\Psi_{\alpha}$ is aggregative. Using simple calculations $\Psi_{\alpha}$ can be written as

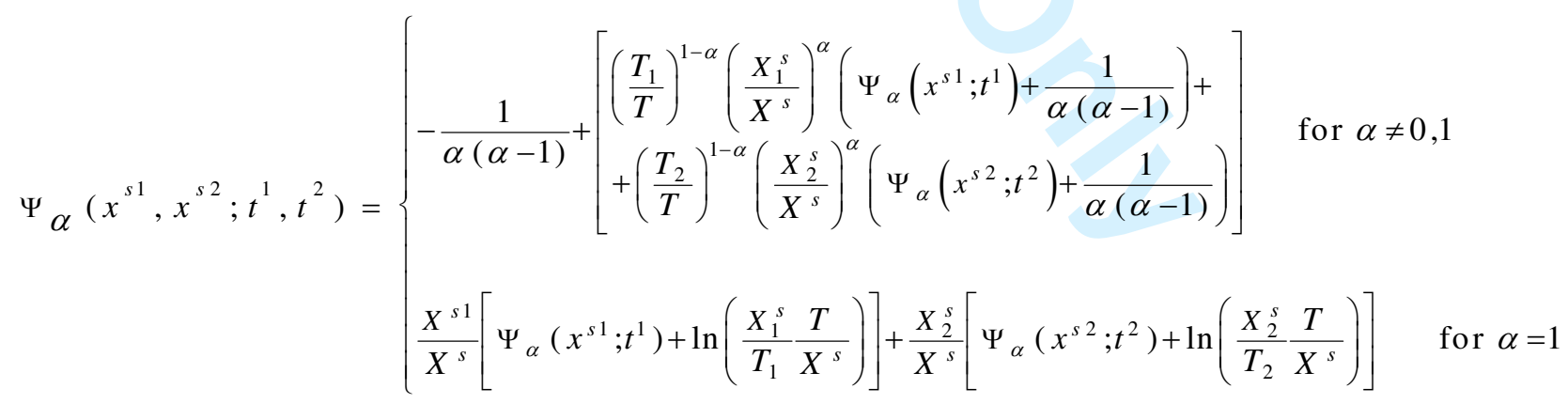


On the other hand, $T=T_{1}+T_{2}$ and $X^{s}=X_{1}^{s}+X_{2}^{s}$. Therefore, $\Psi_{\alpha}$ can be written as $\Psi_{\alpha}\left(x^{s 1}, x^{s 2} ; t^{1}, t^{2}\right)=A\left(\Psi_{\alpha}\left(x^{s 1} ; t^{1}\right), \frac{X_{1}^{s}}{T_{1}}, T_{1}, \Psi_{\alpha}\left(x^{s 2} ; t^{2}\right), \frac{X_{2}^{s}}{T_{2}}, T_{2}\right)$, which completes the proof.

\author{
Appendix B \\ [Insert Table 4 around here] \\ [Insert Table 5 around here] \\ [Insert Figure 3 around here]
}

\title{
References
}

AIGINGER, K. and DAVIES, S.W. (2004) Industrial specialization and geographic concentration: two sides of the same coin? Not for the European Union, Journal of Applied Economics VII(2), 231-248.

AIGINGER, K. and PFAFFERMAYR, M. (2004) The single market and geographic concentration in Europe, Review of International Economics 12(1), 1-11.

ALONSO-VILLAR, O. (2011) Measuring geographic concentration: Lorenz curves and their decompositions, Annals of Regional Science, forthcoming (doi:10.1007/s00168010-0369-5).

ALONSO-VILLAR, O., CHAMORRO-RIVAS, J.M., and GONZÁLEZ-CERDEIRA, X. (2004) Agglomeration economies in manufacturing industries: the case of Spain, Applied Economics 36(18), 2103- 2116.

ALONSO-VILLAR, O. and DEL RÍO, C. (2010a) Local versus overall segregation measures, Mathematical Social Sciences 60, 30-38.

ALONSO-VILLAR, O. and DEL RÍO, C. (2010b) Concentration of economic activity: inequality-based measures, Documento de Traballo 1006, Departamento de Economía Aplicada, Universidade de Vigo. 
AMITI, M. (1999) Specialization patterns in Europe, Weltwirtschaftliches Archiv 135(4), 573-593.

ARBIA, G. (1989): Spatial data configuration in statistical analysis of regional economic and related problems. Kluwer, Dordrecht.

ATKINSON, A.B. (1970) On the measurement of inequality, Journal of Economic Theory 2, 244-263.

BICKENBACH, F. and BODE, E. (2008) Disproportionality measures of concentration, specialization, and localization, International Regional Science Review 31, 359-388.

BOTAZZI, D., DOSI, G., FAGIOLO, G., and SECCHI, A. (2008) Sectoral and geographical specificities in the spatial structure of economic activities, Structural Change and Economic Dynamics 19, 189-202.

BRAKMAN, S., GARRETSEN, H., GORTER, J., VAN DER HORST, A. and SCHRAMM, M. (2005) New economic geography, empirics, and regional policy, CPB (Netherlands Bureau for Economic Policy Analysis) Special Publication 56.

BRÜLHART, M. (2001) Evolving geographical concentration of European manufacturing industries, Weltwirtschaftliches Archiv 137(2), 215-243.

BRÜLHART, M. and TRAEGER, R. (2005) An account of geographic concentration patterns in Europe, Regional Science and Urban Economics 35(6), 597-624.

COMBES, P.-P. and OVERMAN, H. (2004) The spatial distribution of economic activities in the EU, in HENDERSON J.V. and THISSE J.-F. (Eds), Handbook of Regional and Urban Economics 4, pp. 2845-2909. Elsevier-North Holland, Amsterdam.

COVER, T.M. and THOMAS, Y.A. (1991): Elements of Information Theory. Wiley Interscience, New York.

CUTRINI, E. (2009) Using entropy measures to disentangle regional from national localization patterns, Regional Science and Urban Economics 39, 243-250.

CUTRINI, E. (2010) Specialization and concentration from a twofold geographical perspective: evidence from Europe, Regional Studies 44(3), 315-336. 
DUNCAN, O.D. and DUNCAN, B. (1955) A methodological analysis of segregation indexes, American Sociological Review 20(2), 210-217.

DURANTON, G. and OVERMAN, H. (2005) Testing for localization using microgeographic data, Review of Economic Studies 72, 1077-1106.

ELLISON, G. and GLAESER, E. (1997) Geographic concentration in U.S. manufacturing industries: a dartboard approach, Journal of Political Economy 105, 889927.

FOSTER, J.E. (1985): Inequality measurement, in PEYTON JOUND H. (Ed), Fair Allocation, American Mathematical Society. Proceedings of Simposia in Applied Mathematics 33, pp. 31-68, Providence.

FRANKEL, D.M. and VOLIJ, O. (2010) Measuring School Segregation, Journal of Economic Theory, forthcoming (doi: 10.1016/j.physletb.2003.10.071).

GUIMARÃES, P., FIGUEIREDO, O. and WOODWARD, D. (2007) Measuring the localization of economic activity: a parametric approach, Journal of Regional Science 47(4), 753-774.

HAALAND, J., KIND, H., MIDELFART KNARVIK, K., and TORSTENSSON, J. (1999) What determines the economic geography of Europe?, CEPR Discussion Paper 2072.

JAMES, D.R. and TAEUBER, K.E. (1985) Measures of segregation, Sociological Methodology 15, 1-32.

KAPUR, J.N. and KESSAVAN, H.K. (1992) Entropy Optimization Principles with Applications. Academic Press, San Diego.

KRUGMAN, P. (1991) Geography and Trade. MIT Press, Cambridge, Massachusetts.

MARCON, E. and PUECH, F. (2003) Evaluating the geographic concentration of industries using distance-base methods, Journal of Economic Geography 3, 409-428.

MAUREL, F. and SÉDILLOT, B. (1999) A measure of the geographic concentration in French manufacturing industries, Regional Science and Urban Economics 29, 575-604. 
MORA, R. and RUIZ-CASTILLO, J. (2010) A Kullback-Leibler measure of conditional segregation, Working Paper-Economic Series 10-15, Department of Economics, Universidad Carlos III de Madrid.

MORI, T., NISHIKIMI, K., and SMITH, T. (2005) A divergence statistic for industrial localization, The Review of Economics and Statistics 87(4), 635-651.

MULLIGAN, G.F. and SCHMIDT C. (2005) A note on localization and specialization, Growth and Change 36(4), 565-575.

OECD (2007): OECD Science. Technology and Industry. Scoreboard 2007.

PALUZIE, E., PONS, J., and TIRADO, D. (2004) The geographical concentration of industry across Spanish regions, 1856-1995, Review of Regional Research, 24(2), 143160.

PÉREZ-XIMÉNEZ, D. and SANZ-GRACIA, F. (2007) Geographical concentration of service activities across U.S: states and counties. 1969-2000. Paper presented at the XVII International RESER (European Research Network on Services and Space) Conference in Tempere (Finland).

REARDON, S.F. and FIREBAUGH, G. (2002) Measures of multigroup segregation, Sociological Methodology 32, 33-76.

RESMINI, L. (2007) Regional patterns of industry location in transition countries: does economic integration with the European Union matter?, Regional Studies 41(6), 747764.

SHANNON, C.E. (1948) A mathematical theory of communication, The Bell System Technical Journal 27, 379-423, 623-656.

SHORROCKS, A.F. (1984) Inequality decompositions by population subgroups, Econometrica 48, 1369-1386.

SILBER, J. (1992) Occupational segregation indices in the multidimensional case: a note, The Economic Record 68, 276-277. 
SUEDEKUM, J. (2006) Concentration and specialization trends in Germany since reunification, Regional Studies 40(8), 861-873.

WHITE, M.J. (1983) The measurement of spatial segregation, American Journal of Sociology 88 (5), 1008-1018. 


\begin{tabular}{|c|c|c|c|c|}
\hline $\begin{array}{l}\text { Technological groups } \\
2008\end{array}$ & $\begin{array}{c}\text { Contribution to } \\
M \\
(\%)\end{array}$ & $\begin{array}{c}\text { Contribution to } \\
G \\
(\%)\end{array}$ & $\begin{array}{c}\text { Contribution to } \\
\text { IS } \\
(\%)\end{array}$ & $\begin{array}{c}\text { Employment } \\
\text { share } \\
(\%)\end{array}$ \\
\hline High-tech & 12.8 & 8.7 & 8.6 & 5.1 \\
\hline Medium-high-tech & 23.7 & 26.8 & 26.8 & 25.3 \\
\hline Medium-low-tech & 21.3 & 22.1 & 21.4 & 27.2 \\
\hline Low-tech & 42.2 & 42.4 & 43.2 & 42.3 \\
\hline
\end{tabular}

Table 1. Contribution of each group of sectors to overall concentration at a two-digit level and their employment shares. 


\begin{tabular}{|c|c|c|c|}
\hline $\begin{array}{c}\text { Technological groups } \\
2008\end{array}$ & $\Psi_{1}\left(x^{s} ; t\right)$ & $G^{s}$ & $D^{s}$ \\
\hline High-tech & 0.336 & 0.438 & 0.330 \\
\hline Medium-high-tech & 0.101 & 0.250 & 0.190 \\
\hline Medium-low-tech & 0.044 & 0.160 & 0.119 \\
\hline Low-tech & 0.037 & 0.151 & 0.109 \\
\hline
\end{tabular}

Table 2. Partial concentration indexes of each technological group. 


\begin{tabular}{|c|cc|cc|cc|}
\hline & $\begin{array}{c}\text { Concentration/structure } \\
\text { factor decomposition } \\
M\end{array}$ & \multicolumn{2}{|c|}{$\begin{array}{c}\text { Concentration/structure } \\
\text { factor decomposition } \\
G\end{array}$} & \multicolumn{2}{|c|}{$\begin{array}{c}\text { Concentration/structure } \\
\text { factor decomposition } \\
I S\end{array}$} \\
\hline $1977-1981$ & $109.98 \%$ & $-9.98 \%$ & $109.17 \%$ & $-9.17 \%$ & $109.16 \%$ & $-9.16 \%$ \\
\hline $1985-1990$ & $64.21 \%$ & $35.79 \%$ & $72.73 \%$ & $27.27 \%$ & $73.89 \%$ & $26.11 \%$ \\
\hline $2001-2008$ & $59.86 \%$ & $40.14 \%$ & $75.82 \%$ & $24.18 \%$ & $75.34 \%$ & $24.66 \%$ \\
\hline
\end{tabular}

Table 3. Intertemporal decompositions of overall concentration indexes. 
Table 4. Classification of two-digit industries by technological intensity: 1977-1992. 
Table 5. Classification of two-digit industries by technological intensity: $1993-2008 .^{22}$ 


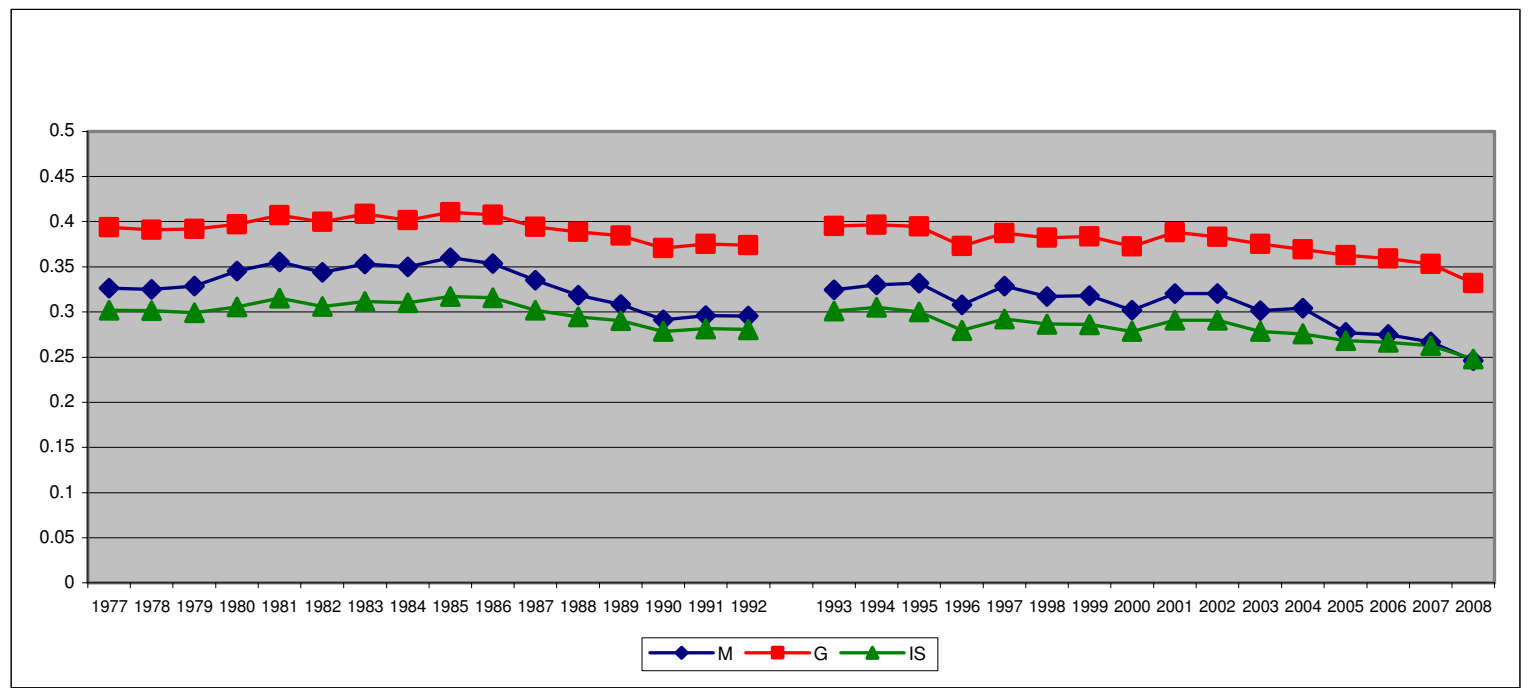

Figure 1. Overall concentration of the manufacturing industry over the period 19772008. 


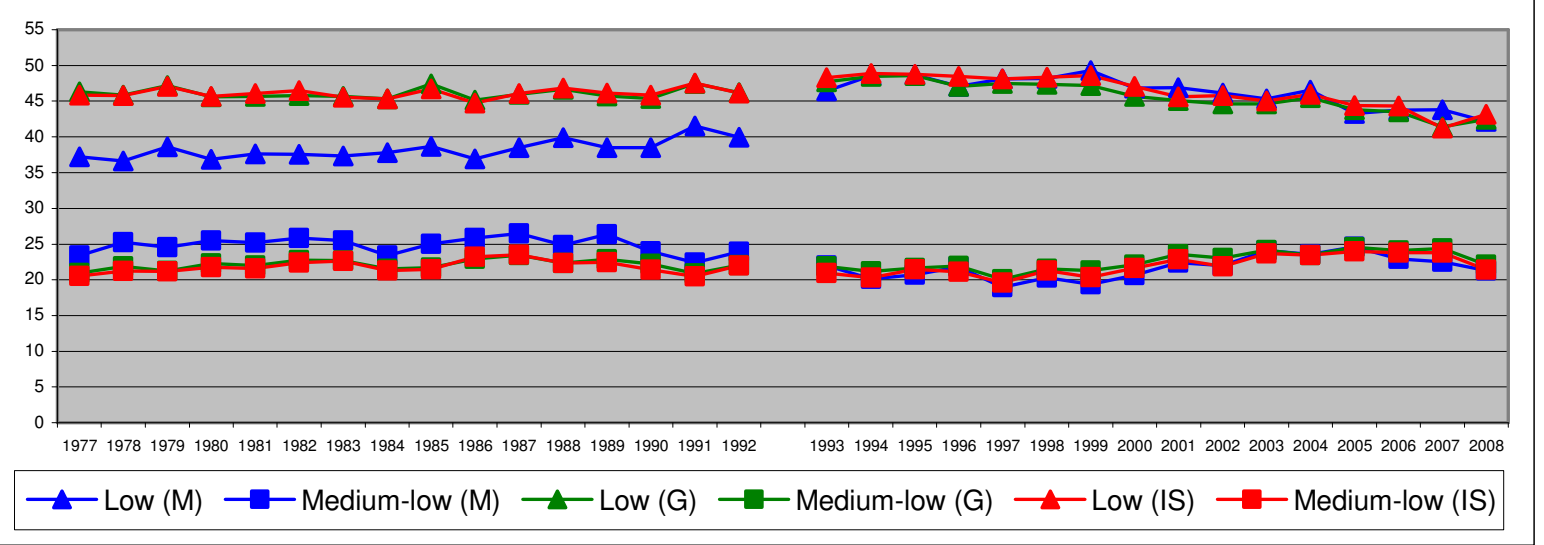

Figure 2. Contribution of each technological group to three overall concentration indexes. 


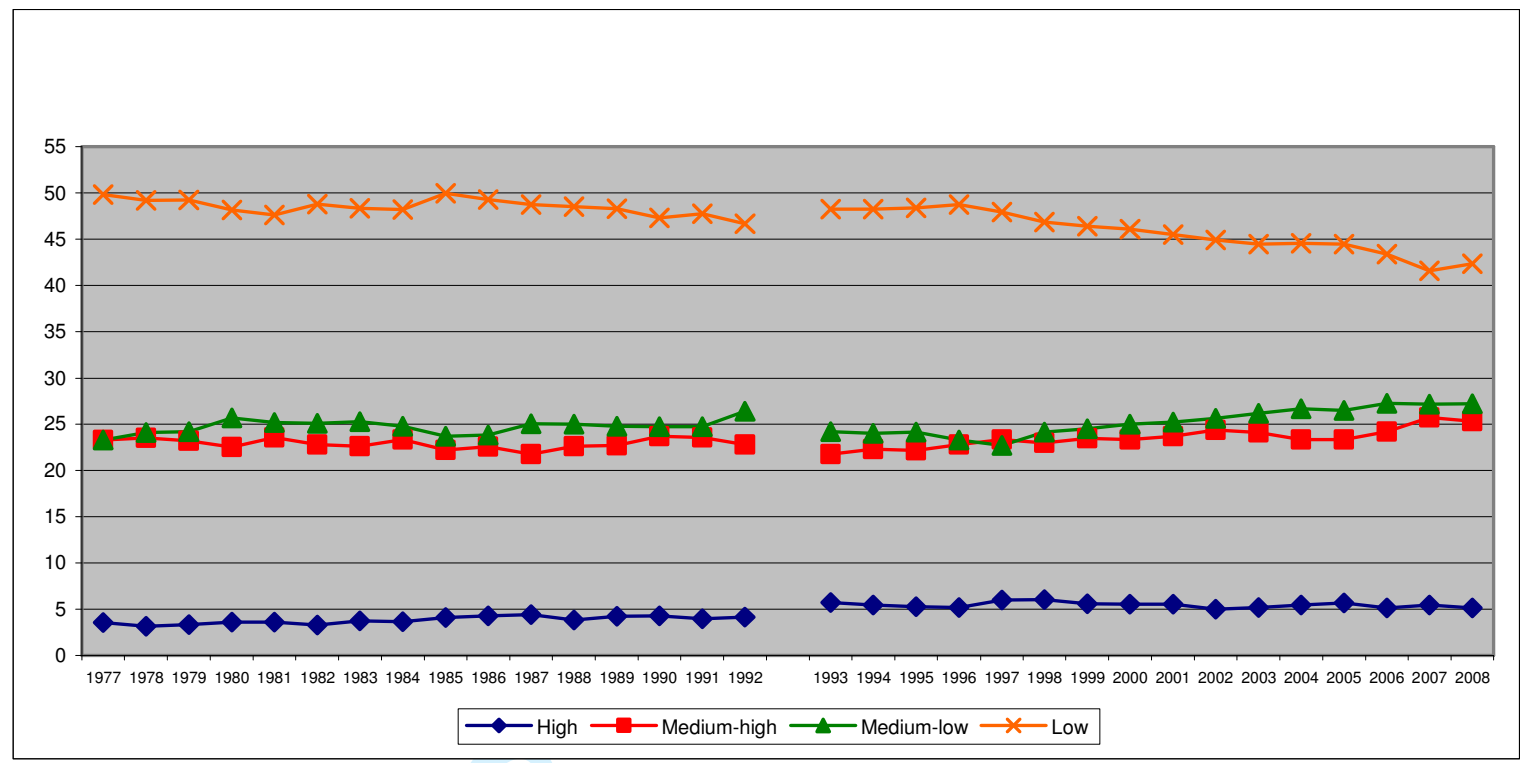

Figure 3. Manufacturing employment share along the period 1977-2008. 


\footnotetext{
${ }^{1}$ Some of these properties have been used in ALONSO-VILLAR (2011) to characterize employment Lorenz curves.
}

2 For an econometric approach to the roles played by natural advantages and technological spillovers, see BOTTAZZI et al. (2008).

${ }^{3}$ The properties of entropy measures outside economics can be seen in SHANNON (1948) and KAPUR and KESAVAN (1992).

4 For other proposals, see BICKENBACK and BODE (2008) and ALONSO-VILLAR and DEL RÍO (2010b).

${ }^{5}$ The GE family also includes $\Psi_{0}\left(x^{s} ; t\right)=\sum_{l} \frac{t_{l}}{T} \ln \left(\frac{t_{l} / T}{x_{l}^{s} / X^{s}}\right)$ if $x_{l}^{s} \neq 0 \quad \forall l$.

${ }^{6}$ The values of these indexes are unbounded.

${ }^{7}$ For technical definitions see Appendix A.

${ }^{8}$ Properties 2 and 4 imply aversion to inequality in distribution $\left(\frac{x_{1}^{s}}{t_{1}}, \ldots, \frac{x_{L}^{s}}{t_{L}}\right)$.

${ }^{9}$ This is related to a property proposed in the literature of income distribution according to which, the greater inequality increase, the lower the income of the poorer individual involved in a regressive transfer from the poorer to the richer (see ATKINSON, 1970). In our context, this means that disequalizing movements of workers between locations lead to higher concentration increases in the sector when these movements occur between locations in which the relative presence of this sector, as compared with overall employment there, is low.

${ }^{10} G^{s}$ and $D^{s}$ take values in the interval $[0,1)$.

${ }^{11}$ For additional properties, see MORA and RUIZ-CASTILLO (2010).

${ }^{12}$ If grouping (say) three-digit industries into two-digit industries, overall concentration at a three-digit level can be expressed as aggregate concentration at a two-digit level (the between-group component) plus the concentration inside each two-digit industry considered separately (the distribution of each three-digit industry is compared with that of the two-digit industry to which it belongs rather than with distribution $t$ ). 
${ }^{13}$ As opposed to the $I S$ index, which is based on comparisons between $\frac{x_{l}^{s}}{X^{s}}$ and $\frac{t_{l}}{T}$ in each location, in the global concentration index proposed by MULLIGAN and SCHMIDT (2005), $G(L)=\sum_{s} \frac{X^{s}}{T}\left(\frac{1}{2} \sum_{l}\left|\frac{x_{l}^{s}}{t_{l}}-\frac{X^{s}}{T}\right|\right), \frac{x_{l}^{s}}{t_{l}}$ is contrasted with $\frac{X^{s}}{T}$.

${ }^{14}$ In fact, the Spearman correlation coefficient between these indexes ranges between 0.94 and 0.98 .

15 The values of these indexes for 2008 are shown in Figure 1, which includes not only these figures but also those of the whole period.

${ }^{16}$ This result is not a consequence of the small weight of the high-tech industry in the economy since the GE family is unaffected by the size of the sector (see property 3 ).

17 The change from 1992 to 1993 is due to use of a different classification of manufacturing industries: From 1977 to 1992, EPA gathered information at a two-digit level according to classification CNAE-1974, while from 1993 to 2008, the classification used was CNAE-93.

18 This finding corroborates that obtained by PALUZIE et al. (2004), who used alternative indexes and datasets for the period 1955-1995.

${ }^{19}$ For arguments in favor of using this method in the case of entropy measures, see BRÜLHART and TRAEGER (2005) and CUTRINI (2010).

${ }^{20}$ Since classification of the manufacturing industry at a two-digit level between 1977 and 1992 is different from the one used between 1993 and 2008, we have used another technological grouping (see Tables 4 and 5 in Appendix B). In any case, a broad criterion similar to that used in 2008 has been used for the whole period.

${ }^{21}$ The case where $\alpha=0$ is discarded, because when the sector has no employment in location $l$ (i.e., when $x_{l}^{s}=0$ ) and $\alpha=0$, the index value would be infinite and, therefore, it makes no sense. The case where $\alpha=1$ does not have the same problem since $\lim _{x_{l} \rightarrow 0} \frac{x_{l}^{s} / X^{s}}{t_{l} / T} \ln \left(\frac{x_{l}^{s} / X^{s}}{t_{l} / T}\right)=0$.

${ }^{22}$ Since in this study industries are considered at a two-digit level and the OECD (2007) and INE classifications consider both two- and three-digit industries, we have introduced some changes with respect to them. In particular, the INE includes one of the subsectors of sector $\mathbf{2 4}$ in the high-tech group and the remaining subsectors in the medium-high-tech group. Here, we have decided to include the whole sector in the latter 
group. On the other hand, the INE classifies part of sector 35 (i.e. aircraft) in the former group and part in the latter. We have decided to include the whole sector in the former. 